\title{
BMJ Open Palivizumab and prevention of childhood respiratory syncytial viral infection: protocol for a systematic review and meta-analysis of breakthrough infections
}

Shelly Jun, ${ }^{1}$ Meghan Sebastianski, ${ }^{1}$ Robin Featherstone, ${ }^{1,2}$ Joan Robinson ${ }^{3}$

To cite: Jun S, Sebastianski M, Featherstone $\mathrm{R}$, et al. Palivizumab and prevention of childhood respiratory syncytial viral infection: protocol for a systematic review and metaanalysis of breakthrough infections. BMJ Open 2019;9:e029832. doi:10.1136/ bmjopen-2019-029832

- Prepublication history and additional material for this paper are available online. To view please visit the journal (http:// dx.doi.org/10.1136/bmjopen2019-029832).

Received 13 February 2019 Revised 6 May 2019 Accepted 26 June 2019

Check for updates

(C) Author(s) (or their employer(s)) 2019. Re-use permitted under CC BY-NC. No commercial re-use. See rights and permissions. Published by BMJ.

${ }^{1}$ Alberta Strategy for PatientOriented Research (SPOR) Knowledge Translation Platform, Department of Pediatrics, University of Alberta, Edmonton, Alberta, Canada

${ }^{2}$ Alberta Research Centre for Health Evidence (ARCHE), Department of Pediatrics, University of Alberta, Edmonton, Alberta, Canada

${ }^{3}$ Division of Pediatric Infectious Diseases, Department of Pediatrics, University of Alberta, Edmonton, Alberta, Canada

Correspondence to

Dr Joan Robinson;

jr3@ualberta.ca

\section{ABSTRACT}

Introduction Childhood respiratory syncytial virus (RSV) infection is a global phenomenon that can lead to fatal respiratory illness. Palivizumab is a drug that is routinely used in affluent countries as a prophylaxis against RSV infection; nevertheless, breakthrough infections are often reported. In light of new findings on potential RSV resistance to palivizumab, an up-to-date synthesis of evidence on effectiveness is needed. Furthering existing reviews, a broadened scope to better reflect effectiveness in a 'real world' clinical context is also important. This systematic review and meta-analysis will enhance our understanding of the effectiveness of palivizumab in varying populations of children. Findings from this review will inform recommendations for best practices regarding palivizumab use for childhood RSV infection as well as research priorities in RSV vaccine development. Methods and analysis We will conduct a systematic review of primary population-based studies that examine the incidence of palivizumab breakthrough infections in children, published between 1997 to present. In collaboration with a research librarian, four electronic databases (MEDLINE, Embase, Cochrane Library, Web of Science) and additional sources will be searched. Study screening and quality assessment will be performed in duplicate. Data will be extracted by one reviewer, with partial and random verification by a second reviewer. The primary outcomes to assess breakthrough RSV infection will be hospitalisation, length of stay and the need for intensive care unit admission and mechanical ventilation in children receiving palivizumab. The secondary outcome will be RSV-associated mortality. We will conduct a meta-analysis using pooled effectiveness data, and include subgroup analyses by patient comorbidities and drug compliance. Sensitivity analyses for risk of bias and study design will also be performed.

Ethics and dissemination This systematic review will only include data from previously published literature and is therefore exempt from ethics approval. Final results will be disseminated through peer-reviewed publication and presented at academic conferences and scientific meetings engaging paediatric researchers and healthcare providers. Should findings from this review necessitate updates to current clinical practice guidelines, we intend to establish a working group to engage relevant health administrators and decision makers.
Strengths and limitations of this study

- Our proposed meta-analysis integrates multiple statistical techniques for a comprehensive evaluation of palivizumab effectiveness.

- Using a two-fold approach to quality assessment, this review considers the quality of both individual study methodology and individual outcomes as a body of evidence.

- This review will provide an up-to-date assessment of palivizumab effectiveness and findings will inform existing and new clinical guidelines.

- Knowing the burden of respiratory syncytial virus (RSV)-related hospital care will support future economic analyses of RSV vaccines.

PROSPERO registration number CRD42019122120.

\section{INTRODUCTION}

Respiratory syncytial virus in children

Human respiratory syncytial virus (RSV) infects almost all children within the first 2 years of life. Typically, RSV infection manifests as the common cold; however, some children develop acute lower respiratory infections (ALRI), a leading cause of childhood morbidity and mortality. ${ }^{1}$ Globally, RSV accounts for approximately 33.1 million annual ALRI episodes in children. ${ }^{2}$ Across North America, the rates of RSV-associated paediatric hospitalisations remain high. ${ }^{3}$ Although supportive care is commonly used to manage RSV symptoms, ${ }^{4}$ no existing treatment for RSV infection has been demonstrated to be effective. ${ }^{5}$

\section{Palivizumab}

Palivizumab is an expensive monoclonal antibody that binds to a RSV surface glycoprotein, the fusion protein and inhibits viruscell membrane fusion; thereby inhibiting 
RSV replication. ${ }^{6}$ Despite its promising mechanism of action, the clinical evidence for palivizumab is limited to demonstrated effectiveness within select population groups in developed countries, where palivizumab is affordable and available. ${ }^{8}$ Moreover, some animal and human studies have demonstrated RSV resistance to palivizumab. ${ }^{9}{ }^{10}$ In an effort to mitigate the burden of childhood RSV, attention has been directed towards preventative strategies. Current clinical recommendations from the American Academy of Paediatrics (AAP), which may differ from other jurisdictions, indicate for all high risk infants to receive palivizumab: preterm infants with chronic lung disease (CLD), preterm infants without CLD of prematurity or congenital heart disease (CHD), infants with haemodynamically significant CHD, children with anatomic pulmonary abnormalities or neuromuscular disorder and profoundly immunocompromised children. ${ }^{11}$

\section{Why is it important to do this review?}

To date, there have been two reviews on the effectiveness of palivizumab ${ }^{12}{ }^{13}$; however, both are limited in scope. The 2013 review includes only randomised controlled trials, ${ }^{12}$ and synthesised findings from a very small number of studies. The 2014 review excluded prospective studies and registries with end dates in 2013. ${ }^{13}$ Moreover, both existing reviews limited the study population to AAP-defined high risk infants. Care of infants with prematurity has changed remarkably since the original palivizumab trials were conducted with far less use of invasive ventilation and complete repair of CHD now occurs at a much younger age. Therefore, recent retrospective studies are of potential value to assessing real-world effectiveness in the modern era. At this time, an updated evidence base of palivizumab effectiveness with an inclusive paediatric population is needed. Given the evidence for potential resistance to palivizumab, ${ }^{10}$ there is a need to determine whether there is new evidence demonstrating decreasing effectiveness over time. This evidence is important to justify continued use of palivizumab for the current indications, or to inform changes to clinical guidelines and practice. Coupled with its implications on health outcomes, confirming palivizumab effectiveness is also foundational to a real assessment of economic benefit and implications for emerging RSV vaccines.

\section{Objective}

To evaluate the incidence of palivizumab breakthrough RSV infections in children.

\section{METHODS AND ANALYSIS}

This systematic review protocol has been registered with the PROSPERO International Prospective Register of Systematic Reviews (http://www.crd.york.ac.uk/prospero), as CRD42019122120. We prepared this protocol in accordance with the Preferred Reporting Items for
Systematic Review and Meta-analysis Protocol (PRISMA-P) checklist. ${ }^{14}$

\section{Study design}

We will undertake a PRISMA-compliant ${ }^{15}$ systematic review and meta-analysis guided by the Cochrane Collaboration and Centre for Reviews and Dissemination.

\section{Inclusion and exclusion criteria}

Types of studies

We will include primary population-based research studies that report on the effectiveness of palivizumab use for prevention of RSV-confirmed hospitalisation in children (ie, randomised controlled trials, cohort studies and case-control studies) and will screen the reference lists of systematic reviews identified by the search for relevant studies. Full-text studies from databases and other sources will be included, however abstracts without full text will be excluded. There will be no language restrictions. We will include studies published from 1997 to present, as marketing of palivizumab for paediatric prophylaxis was approved by the US Federal Drug Administration in June 1998.

\section{Population}

Studies on children who received palivizumab for any indication will be included. Infants or children with cystic fibrosis will be excluded as a recent RSV systematic review was published on this population. ${ }^{16}$

\section{Intervention/comparison}

We will include studies that report the effectiveness of palivizumab to prevent RSV hospitalisation, with or without a comparison group. Studies that include historical RSV cohorts for comparison will be treated as a single arm study, as RSV hospitalisation rates vary from year to year. We will exclude studies that involve palivizumab use for RSV outbreak control in hospitals and studies that retrospectively determine palivizumab use from a population of patients hospitalised for RSV infection.

\section{Outcome measures}

Primary outcomes measures will include hospitalisation due to RSV, hospital length of stay, intensive care unit (ICU) admission, ICU length of stay and the need for mechanical ventilation. The secondary outcome will be mortality due to RSV infection.

\section{Search methods}

\section{Electronic databases}

We will search the following electronic databases: Ovid MEDLINE (1947-), Ovid Embase (1974-), Wiley Cochrane Library (inception-) and Web of Science (All databases) via Clarivate Analytics (1864-). Our search strategy will combine index terms (eg, MeSH) and text words for palivizumab, or monoclonal antibodies for RSV. The MEDLINE search strategy will be peer-reviewed by a second research librarian and then translated into additional databases. Search results will be limited to human 
studies published since 1997. No language restrictions will be applied and any studies included based on their English title and/or abstract will be translated. See online supplementary appendix A for the MEDLINE search strategy.

\section{Additional sources}

We will also search the trial registry ClinicalTrials.gov for relevant drug trials on palivizumab, and the regulatory agency website Drugs@FDA for unpublished reports. Conference proceedings will be included in our search of Embase and Web of Science (which includes the Conference Proceedings Citation Index). Finally, we will hand-search reference lists of relevant systematic reviews and included studies. Search results will be exported to Endnote $\mathrm{X} 7$ for primary screening.

\section{Data collection}

Study selection

Two review authors will independently screen all studies identified from the search in two phases, primary and secondary screening, assessing study eligibility using predetermined inclusion and exclusion criteria. During primary screening, the authors will review the title and abstracts of all unique records retrieved during the search using EndNote X7, and classify each as 'include/unsure' or 'exclude'. For secondary screening, full texts of all 'include/unsure' records will be retrieved and the two authors will independently review and select studies that meet the inclusion criteria. If disagreements between the authors cannot be resolved by discussion, a third review author will be consulted.

\section{Data extraction}

Data from included studies will be extracted using piloted and standardised electronic data forms (Microsoft Excel 2010) by one author, with partial verification by a second author to ensure accuracy and completeness. Discrepancies will be resolved through discussion or third party consultation.

The data extraction form will capture study characteristics (ie, publication year, study design, funding support, sample size, palivizumab prophylaxis regimen), patient characteristics (ie, age, sex, comorbid disease), type of RSV testing performed and outcomes (ie, RSV-confirmed hospital admission, length of stay, need for ventilation, ICU admission and RSV-associated mortality).

\section{Data analysis and synthesis}

\section{Primary analyses}

Descriptive analysis will be used for study and patient characteristics. Pooled effectiveness data will undergo meta-analysis in RevMan (RevMan V.5.3 Cochrane) using relative risk ratios for binary events and mean differences for continuous data. Due to expected variations between the included studies we will use a random effects model and heterogeneity will be measured using the $\mathrm{I}^{2}$ statistic, with values greater than $25 \%, 50 \%$ and $75 \%$ considered moderate, high and very high heterogeneity, respectively.
If possible publication bias will be determined using a funnel plot and Egger's test. ${ }^{17}$

\section{Secondary analyses}

If appropriate with the generated data, we will calculate the number needed to treat, using pooled risk ratios and ranges of control event rates from our primary analysis, to enhance the clinical meaningfulness of our report.

\section{Subgroup and sensitivity analyses}

Subgroup analyses will be conducted based on patient groups of clinical interest and intervention compliance. The following subgroups have been identified: preterm infants, children with CLD, children with haemodynamically significant CHD, mixed risk population (data were not divided according to a specific risk) and two subgroups based on compliance with the dosing schedule-children who received either $<80 \%$ or $\geq 80 \%$ of the recommended number of palivizumab doses.

We will perform sensitivity analyses based on study design (ie, prospective vs retrospective), type of RSV testing performed and risk of bias (see section 'Quality Assessment').

\section{Missing data}

For the meta-analysis, we will calculate missing parameters from the provided data if possible and exclude studies that do not report or provide enough information to calculate effect estimates. We will not attempt to contact authors regarding missing or unreported data.

\section{Quality assessment}

Individual studies

Two authors will independently assess the methodological quality of each primary study based on the study design. For randomised control trials we will apply the Cochrane Risk of Bias tool, using the high, low or unclear categories to define risk. ${ }^{18}$ For cohort and case-control studies we will apply the Newcastle-Ottawa Scale with a score of 3 or less considered poor quality with a high risk of bias, a score between 4 and 6 considered fair quality with a medium risk of bias and a score of 7 or greater considered good quality with a low risk of bias. ${ }^{19}$ Discrepancies will be resolved through discussion or third party consultation. Analyses will be informed by assessment of risk of bias, and when necessary, we will down-weigh studies with high risk of bias. ${ }^{20}$

\section{Body of evidence}

Two authors will independently assess the certainty of the body of evidence for each outcome using the Grading of Recommendations Assessment, Development and Evaluation (GRADE) tool using direction from the GRADE Handbook. ${ }^{21}$ Assessments will be made over five domains: risk of bias, inconsistency, indirectness, imprecision and publication bias, with quality scored as high, moderate, low or very low. 


\section{Ethics and dissemination}

There is no ethics protocol to be approved or reported. For methodological transparency, our protocol will be submitted for peer-reviewed publication; and likewise, our final manuscript with study data will be disseminated through peer-reviewed publication. Study findings will be presented at academic conferences and scientific meetings engaging researchers in the field and paediatric healthcare providers. If findings from this review necessitate updates to current clinical practice guidelines, we plan to establish a clinician working group to develop an evidence-based report targeted to health administrators and decision makers.

\section{DISCUSSION}

This systematic review will address a priority health topic, childhood RSV infection, and contribute to the advancement of evidence-based management practices. Based on studies to date, the evidence for palivizumab effectiveness in different subgroups after licensure remains unclear. $^{22-24}$ Moreover, prior reviews have excluded retrospective studies in their analyses of palivizumab effectiveness. ${ }^{12} 13$ This exclusion limits our present understanding of palivizumab, as retrospective studies provide valuable 'real world' data that is often not captured with controlled trials. Our proposed systematic review and meta-analysis will build a comprehensive evidence base on palivizumab effectiveness in children, taking into consideration clinically important 'real world' factors, such as patient comorbidities and compliance. Findings from this review will inform clinical decision-making with regards to expanded indications for palivizumab prophylaxis, and potentially guide vaccination strategies.

\section{Potential limitations}

We anticipate potential limitations to this review. Methods for RSV testing have rapidly evolved since palivizumab was introduced in 1998. As such, in recent years, molecular assays used to detect RSV antigen have greater sensitivity, ${ }^{25}$ which may lead to an underestimation of breakthrough RSV admission in earlier studies. In contrast, some 'Choosing Wisely' campaigns suggest that routine viral testing is not indicated when children are admitted with lower respiratory tract infections, potentially underestimating the number of breakthrough admissions in more recent studies.

\section{CONCLUSION}

RSV is the most common cause of paediatric respiratory hospitalisations. This systematic review of the available evidence on the effectiveness of palivizumab has the potential to change how palivizumab is used and may influence the economic evaluation of RSV vaccines.

\section{AMENDMENTS}

Amendments to the protocol will be documented and reported in PROSPERO, detailing the changes made, date, timing within review conduct and purpose. All amendments will be reported in the final manuscript.

Acknowledgements We would like to thank Sholeh Rahman and Jocelyn Shulhan-Kilroy for their assistance in finalising the inclusion and exclusion criteria.

Contributors SJ drafted the body of the protocol with assistance from MS. RF developed and executed the search strategy and wrote the search section of the protocol manuscript. JR conceived the project and was aided by MS and RF in design of the review. All authors read, revised and approved the final version of the protocol.

Funding This work is supported by the Alberta Strategy for Patient-Oriented Research (SPOR) SUPPORT Unit Knowledge Translation Platform, which is funded by Alberta Innovates and the Canadian Institutes of Health Research.

Competing interests None declared.

Patient consent for publication Not required.

Provenance and peer review Not commissioned; externally peer reviewed.

Open access This is an open access article distributed in accordance with the Creative Commons Attribution Non Commercial (CC BY-NC 4.0) license, which permits others to distribute, remix, adapt, build upon this work non-commercially, and license their derivative works on different terms, provided the original work is properly cited, appropriate credit is given, any changes made indicated, and the use is non-commercial. See: http://creativecommons.org/licenses/by-nc/4.0/.

\section{REFERENCES}

1. Liu L, Oza S, Hogan D, et al. Global, regional, and national causes of under-5 mortality in 2000-15: an updated systematic analysis with implications for the Sustainable Development Goals. Lancet 2016;388:3027-35

2. Shi T, McAllister DA, O'Brien KL, et al. Global, regional, and national disease burden estimates of acute lower respiratory infections due to respiratory syncytial virus in young children in 2015: a systematic review and modelling study. Lancet 2017;390:946-58.

3. Mitchell I, Defoy I, Grubb E. Burden of respiratory syncytial virus hospitalizations in Canada. Can Respir J 2017;2017:1-9.

4. Garzon LS, Wiles L. Management of respiratory syncytial virus with lower respiratory tract infection in infants and children. AACN Clin Issues 2002;13:421-30.

5. Turner TL, Kopp BT, Paul G, et al. Respiratory syncytial virus: current and emerging treatment options. Clinicoecon Outcomes Res 2014;6:217-25.

6. Huang K, Incognito L, Cheng X, et al. Respiratory syncytial virusneutralizing monoclonal antibodies motavizumab and palivizumab inhibit fusion. J Virol 2010;84:8132-40.

7. Johnson S, Oliver C, Prince GA, et al. Development of a humanized monoclonal antibody (MEDI-493) with potent in vitro and in vivo activity against respiratory syncytial virus. $J$ Infect Dis 1997;176:1215-24.

8. Hu J, Robinson JL. Treatment of respiratory syncytial virus with palivizumab: a systematic review. World J Pediatr 2010;6:296-300.

9. Papenburg J, Carbonneau J, Hamelin MÈ, et al. Molecular evolution of respiratory syncytial virus fusion gene, Canada, 2006-2010. Emerg Infect Dis 2012;18:120-4.

10. Zhao X, Chen FP, Megaw AG, et al. Variable resistance to palivizumab in cotton rats by respiratory syncytial virus mutants. $J$ Infect Dis 2004;190:1941-6.

11. American Academy of Pediatrics Committee on Infectious DiseasesAmerican Academy of Pediatrics Bronchiolitis Guidelines Committee. Updated guidance for palivizumab prophylaxis among infants and young children at increased risk of hospitalization for respiratory syncytial virus infection. Pediatrics 2014;134:e62 0-e638.

12. Andabaka T, Nickerson JW, Rojas-Reyes MX, et al. Monoclonal antibody for reducing the risk of respiratory syncytial virus infection in children. Cochrane Database Syst Rev 2013;4:Cd006602.

13. Wegzyn C, Toh LK, Notario G, et al. Safety and effectiveness of palivizumab in children at high risk of serious disease due to respiratory syncytial virus infection: a systematic review. Infect Dis Ther 2014;3:133-58.

14. Shamseer L, Moher D, Clarke M, et al. Preferred reporting items for systematic review and meta-analysis protocols (PRISMA-P) 2015: elaboration and explanation. BMJ 2015;350:g7647. 
15. Moher D, Liberati A, Tetzlaff J, et al. Preferred reporting items for systematic reviews and meta-analyses: the PRISMA statement. Int $J$ Surg 2010;8:336-41.

16. Kua KP, Lee SWH, Swh L. Systematic review of the safety and efficacy of palivizumab among infants and young children with cystic fibrosis. Pharmacotherapy 2017;37:755-69.

17. Egger M, Davey Smith G, Schneider M, et al. Bias in meta-analysis detected by a simple, graphical test. BMJ 1997;315:629-34.

18. Higgins JP, Altman DG, Gøtzsche PC, et al. The Cochrane Collaboration's tool for assessing risk of bias in randomised trials. BMJ 2011;343:d5928.

19. Wells G, Shea B, O'Connell J, et al. The Newcastle-Ottawa scale (NOS) for assessing the quality of nonrandomised studies in metaanalysis. http://www.ohri.ca/programs/clinical_epidemiology/oxford. asp (Accessed 28 Mar 2018).

20. Hartling L, Hamm MP, Fernandes RM, et al. Quantifying bias in randomized controlled trials in child health: a meta-epidemiological study. PLoS One 2014;9:e88008.
21. Schünemann $H J$, Schünemann $A H, O x m a n ~ A D$, et al. Grading quality of evidence and strength of recommendations for diagnostic tests and strategies. BMJ 2008;336:1106-10.

22. Chang SG, Park MS, Yu JE. Outcomes of palivizumab prophylaxis for respiratory syncytial virus infection in preterm children with bronchopulmonary dysplasia at a single hospital in Korea from 2005 to 2009. J Korean Med Sci 2010;25:251-6.

23. Henckel E, Luthander J, Berggren E, et al. Palivizumab prophylaxis and hospitalization for respiratory syncytial virus disease in the Stockholm infant population, 1999 through 2002. Pediatr Infect Dis J 2004;23:27-31.

24. Kusuda S, Koizumi T, Sakai T, et al. Results of clinical surveillance during the Japanese first palivizumab season in 2002-2003. Pediatr Int 2006;48:362-8.

25. Rabon-Stith KM, McGuiness CB, Saunders B, et al. Laboratory testing trends for respiratory syncytial virus, 2007-2011. J Clin Virol 2013;58:575-8 